SLAC-PUB-12006

July 2006

\title{
INVERSE COMPTON SCATTERING ON SOLAR PHOTONS, HELIOSPHERIC MODULATION, AND NEUTRINO ASTROPHYSICS
}

\author{
IgOR V. MOSKALENKO ${ }^{1}$ \\ Hansen Experimental Physics Laboratory, Stanford University, Stanford, CA 94305 \\ Troy A. Porter \\ Santa Cruz Institute for Particle Physics, University of California, Santa Cruz, CA 95064 \\ AND \\ SETH W. DigeL ${ }^{1}$ \\ Stanford Linear Accelerator Center, 2575 Sand Hill Road, Menlo Park, CA 94025 \\ Draft version July 23, 2006
}

\begin{abstract}
We study the inverse Compton scattering of solar photons by Galactic cosmic-ray electrons. We show that the $\gamma$-ray emission from this process is significant with the maximum flux in the direction of the Sun; the angular distribution of the emission is broad. This previously neglected foreground should be taken into account in studies of the diffuse Galactic and extragalactic $\gamma$-ray emission. Furthermore, observations by GLAST can be used to monitor the heliosphere and determine the electron spectrum as a function of position from distances as large as Saturn's orbit down to close proximity of the Sun, thus enabling studies of solar modulation in the most extreme case. This paves the way for the determination of other Galactic cosmic-ray species, primarily protons, near the solar surface leading to accurate predictions of $\gamma$-rays from $p p$-interactions in the solar atmosphere. These albedo $\gamma$-rays will be observable by GLAST, allowing the study of deep atmospheric layers, magnetic field(s), and cosmicray cascade development. The latter is necessary to calculate the neutrino flux from $p p$-interactions at higher energies $(>1 \mathrm{TeV})$. The corresponding neutrino flux from the Sun can be used as a "standard candle" for upcoming $\mathrm{km}^{3}$ neutrino detectors, such as IceCube. Since the solar core is opaque for very high-energy neutrinos, it may be possible to directly study the mass distribution of the Sun.

Subject headings: elementary particles - Sun: general - Sun: interior - Sun: X-rays, gamma rays - cosmic rays - gamma-rays: theory
\end{abstract}

\section{INTRODUCTION}

Interactions of Galactic cosmic-ray (CR) nuclei with the solar atmosphere have been predicted to be a source of very high energy (VHE) neutrinos (Moskalenko et al. 1991; Seckel et al. 1991; Ingelman \& Thunman 1990), and $\gamma$-rays (Seckei et ai. 1991). They are the decay products of chafged and ne itral pions produced in interactions of $\mathrm{CR}$ nucleons with gas. The predictions for these albedo $\gamma$-rays give an integral flux $F_{\gamma}(>100 \mathrm{MeV}) \sim$ $(0.2-0.7) \times 10^{-7} \mathrm{~cm}^{-2} \mathrm{~s}^{-1}$, while analysis of EGRET $\gamma$-ray telescope data has yielded only the upper limit $F_{\gamma}(>100 \mathrm{MeV})=2.0 \times 10^{-7} \mathrm{~cm}^{-2} \mathrm{~s}^{-1}$ (Thompson et al. 1997).

Cosmic-ray electrons comprise $\sim 1 \%$ of the total CR Hux. However, they propagate over the heliospheric volume which is large compared to the solar atmosphere where the albedo $\gamma$-rays are produced. The Sun emits photons which are targets for inverse Compton (IC) scattering by $\mathrm{CR}$ electrons. As a result the heliosphere is a diffuse source of $\gamma$-rays with a broad angular distribution. In this paper, we evaluate the importance of IC scattering within the heliosphere, and discuss the consequences of its measurement by such instruments as the upcoming Gamma Ray Large Area Space Telescope (GLAST) mission. In the following, we use units $\hbar=c=m_{e}=1$.

1 Also Kavli Institute for Particle Astrophysics and Cosmology, Stanford University, Stanford, CA 94309

\section{ANISOTROPIC INVERSE COMPTON SCATTERING IN THE HELIOSPHERE}

The distribution of CR electrons within the heliosphere is isotropic. However, the distribution of solar photons is distinctly anisotropic, with the photons propagating outward from the Sun. The expression for the IC production spectrum for an arbitrary photon angular distribution is given by eq. (8) from Moskalenko \& Strong (2000):

$$
\begin{aligned}
& \frac{d R\left(\gamma_{e}, \epsilon_{1}\right)}{d \epsilon_{2}}=\frac{\pi r_{e}^{2}}{\epsilon_{1}\left(\gamma_{e}-\epsilon_{2}\right)^{2}} \int_{\Omega_{\nu}} d \Omega_{\nu} Q_{\nu}\left(\Omega_{\nu}\right) \\
& \quad \times\left[2-2 \frac{\epsilon_{2}}{\gamma_{e}}\left(\frac{1}{\epsilon_{1}^{\prime}}+2\right)+\frac{\epsilon_{2}^{2}}{\gamma_{e}^{2}}\left(\frac{1}{\epsilon_{1}^{\prime 2}}+2 \frac{1}{\epsilon_{1}^{\prime}}+3\right)-\frac{\epsilon_{2}^{3}}{\gamma_{e}^{3}}\right]
\end{aligned}
$$

where $r_{e}$ is the classical electron radius, $\gamma_{e}$ is the electron Lorentz-factor, $\epsilon_{1}$ and $\epsilon_{1}^{\prime}$ are the energies of the background photon in the laboratory system (LS) and electron rest system, correspondingly, $\epsilon_{2}$ is the LS energy of upscattered photon, $\epsilon_{2} \leq 2 \gamma_{e} \epsilon_{1}^{\prime} /\left(1+2 \epsilon_{1}^{\prime}\right), \epsilon_{1}^{\prime}=$ $\epsilon_{1} \gamma_{e}\left(1+\beta_{e} \cos \zeta\right), \zeta$ is the LS angle between the momenta of the electron and incoming photon $(\zeta=0$ for head-on collisions, see Fig. 1), $\epsilon_{2 \max }=4 \epsilon_{1} \gamma_{e}^{2} /\left(1+4 \epsilon_{1} \gamma_{e}\right)$ is the maximal energy of the upscattered photons, and $Q_{\nu}\left(\Omega_{\nu}\right)$ is the normalised angular distribution of target photons at a particular spatial point $\left(\int d \Omega_{\nu} Q_{\nu}=1\right)$.

The $\gamma$-ray flux as a function of $\theta$ (see Fig. 1) can be calculated by integrating eq. (1) along the limt-of-sight taking into account the distribution of electrons and solar 


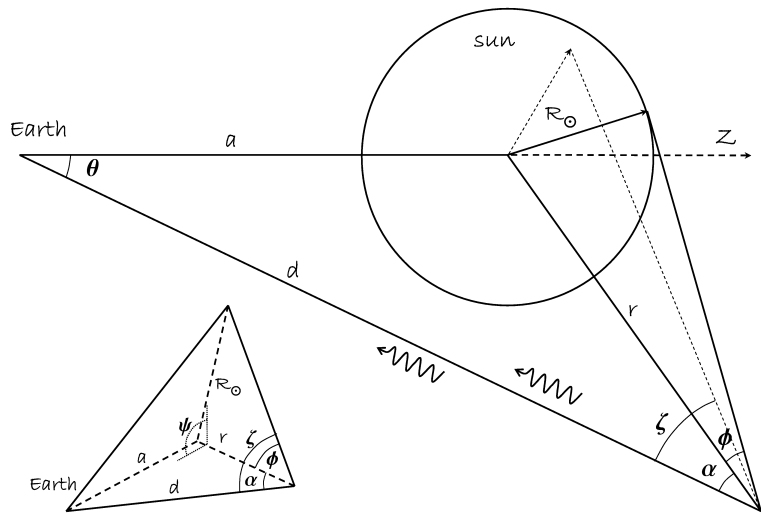

FIG. 1.- Variables involved in the calculation. The large plot shows a 2-dimensional sketch; the dotted lines are a projection of the 3 -dimensional angles. The inset defines the angles in 3 dimensions.

photons:

$$
\begin{aligned}
\frac{d F_{\gamma}}{d \epsilon_{2}}=\frac{1}{2} \int_{L} d x \frac{R_{\odot}^{2}}{r^{2}} & \int d \gamma_{e} \frac{d J_{e}\left(r, \gamma_{e}\right)}{d \gamma_{e}} \\
& \times \int d \epsilon_{1} \frac{d n_{b b}\left(\epsilon_{1}, T_{\odot}\right)}{d \epsilon_{1}} \frac{d R\left(\gamma_{e}, \epsilon_{1}\right)}{d \epsilon_{2}}
\end{aligned}
$$

where the factor $1 / 2$ takes into account that only half of the blackbody photons are moving outward from the solar surface, $J_{e}\left(r, \gamma_{e}\right)$ is the electron intensity, $r$ is the radial distance from the Sun, $R_{\odot}$ is the solar radius, and $d n_{b b}\left(\epsilon_{1}, T_{\odot}\right) / d \epsilon_{1}$ is a blackbody distribution at temperature $T_{\odot}$.

Close to the Sun, $Q_{\nu}\left(\Omega_{\nu}\right)$ is given by

$$
\begin{aligned}
& Q_{\nu}(r, \phi)=\frac{1}{\pi P(r)}\left(1-\frac{r^{2}}{R_{\odot}^{2}} \sin ^{2} \phi\right), \\
& P(r)=1-\frac{r^{2}-R_{\odot}^{2}}{2 r R_{\odot}} \ln \left(\frac{r+R_{\odot}}{r-R_{\odot}}\right), \\
& \cos \zeta=\cos \alpha \cos \phi+\sin \alpha \sin \phi \cos \psi,
\end{aligned}
$$

where $\int_{0}^{2 \pi} d \psi \int_{\cos \phi^{\max }}^{1} Q_{\nu}(r, \phi) d \cos \phi=1, \sin \phi^{\max }=$ $R_{\odot} / r$. For sufficiently large distances, this reduces to a delta function, $Q_{\nu}(\theta)=\delta(\cos \zeta-\cos \alpha)$, where $\cos \alpha=$ $(d-a \cos \theta) /\left(a^{2}+d^{2}-2 a d \cos \theta\right)^{1 / 2}, a=1 \mathrm{AU}$, and the variables are illustrated in Fig. [1] For $r \gtrsim 20 R_{\odot}$ the delta function approximation can be used.

The IC $\gamma$-ray flux essentially falls as $1 / r$ with heliocentric distance (eq. 2]). Therefore, it is straightforward to estimate the region which contributes most to the IC emission. The inner boundary (in AU) is given by

$$
r_{1}= \begin{cases}\sin \theta, & \theta<90^{\circ}, \\ 1, & \theta \geq 90^{\circ}\end{cases}
$$

Approximately $90 \%$ of the total $\gamma$-ray flux is produced between $r_{1}$ and $r_{2}=10 r_{1}$ (Figure 2).

The electron spectrum as a function of position in the heliosphere is obtained by adopting the force-field approximation and deriving the radial dependence of the modulation potential using a toy model. This should be sufficient, as we seek only to illustrate the effect, and are interested in electron energies above $\sim 5 \mathrm{GeV}$ where the heliospheric modulation is moderate. Assuming a separability of the heliospheric diffusion coefficient

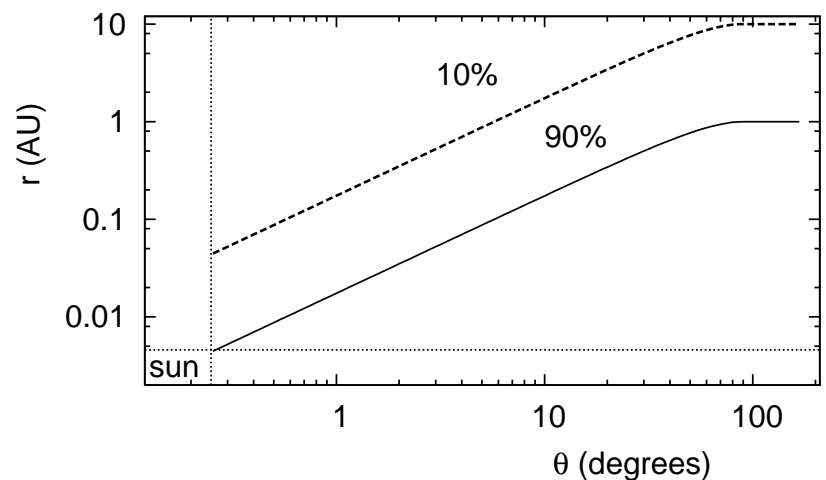

FIG. 2.- The range of heliospheric distances probed by the IC $\gamma$-rays versus angular distance from the Sun. $90 \%$ of the predicted $\gamma$-ray flux is produced in the region between the solid and dashed lines. Dotted lines show the solar size.

$\kappa=\beta \kappa_{1}(r, t) \kappa_{2}(\gamma, t)$, the modulation potential can be obtained (Gleeson \& Axford 1968):

$$
\Phi(r, t)=\int_{r}^{r_{b}(t)} d x \frac{V(x, t)}{3 \kappa_{1}(x, t)},
$$

where $t$ is the time variable, $r_{b}$ is the heliospheric boundary, and $V$ is the solar wind speed. Assuming a radial dependence of $\kappa_{1} \propto r^{\delta}$, and $V=$ const, yields:

$$
\Phi(r, t) \propto r^{-\delta+1}-r_{b}^{-\delta+1} .
$$

Fuiii \& McDonald (2005) have analysed the radial dependence of the mean free path, $\lambda$, during the solar minima of Cycles 20/22 and Cycle 21 using the data from the IMP 8, Voyagers $1 / 2$, and Pioneer 10 spacecraft. For Cycles 20/22 they obtain a parameterisation $\lambda \propto r^{1.4}$ in the outer heliosphere and $\lambda \propto r^{1.1}$ in the inner heliosphere, where the break is at 10-15 AU. For Cycle 21, a $r^{1.1}$ dependence fits well for both the inner and outer heliosphere. Using these parameterisations, $\kappa_{1} \propto \lambda$, yields (Cycles 20/22)

$$
\Phi_{1}(r)=\frac{\Phi_{0}}{1.88} \begin{cases}r^{-0.4}-r_{b}^{-0.4}, & r>r_{0}, \\ 0.24+8\left(r^{-0.1}-r_{0}^{-0.1}\right), & r<r_{0},\end{cases}
$$

where $\Phi_{0}$ is the modulation potential at $1 \mathrm{AU}, r_{0}=$ $10 \mathrm{AU}, r_{b}=100 \mathrm{AU}$, and we have neglected the time dependence. For Cycle 21 we have

$$
\Phi_{2}(r)=\Phi_{0}\left(r^{-0.1}-r_{b}^{-0.1}\right) /\left(1-r_{b}^{-0.1}\right) .
$$

\section{CALCULATIONS}

The local interstellar CR electron spectrum is approximated by $d J_{e}\left(r_{b}, \epsilon_{e}\right) / d \epsilon_{e}=2 \times 10^{5}\left(\epsilon_{e}+400\right)^{-3.22}$ $\mathrm{cm}^{-2} \mathrm{~s}^{-1} \mathrm{sr}^{-1} \mathrm{MeV}^{-1}$, where $\epsilon_{e}$ is the electron energy in $\mathrm{MeV}$. This expression matches the interstellar local electron spectrum above $\sim 500 \mathrm{MeV}$ as calculated by GALPROP in plain diffusion and reacceleration models (Ptuskin et al. 2006). The solar photospheric temperature is taken as $T=6520 \mathrm{~K}$ (Livingston 1999).

Figure 3 shows the integral intensity above $100 \mathrm{MeV}$ and $1 \mathrm{GeV}$ versus the angular distance from the Sun for no modulation, and for modulated electron spectra according to eqs. (8) and (9) with two modulation levels $\Phi_{0}=500,1000 \mathrm{MV}$; these correspond approximately 


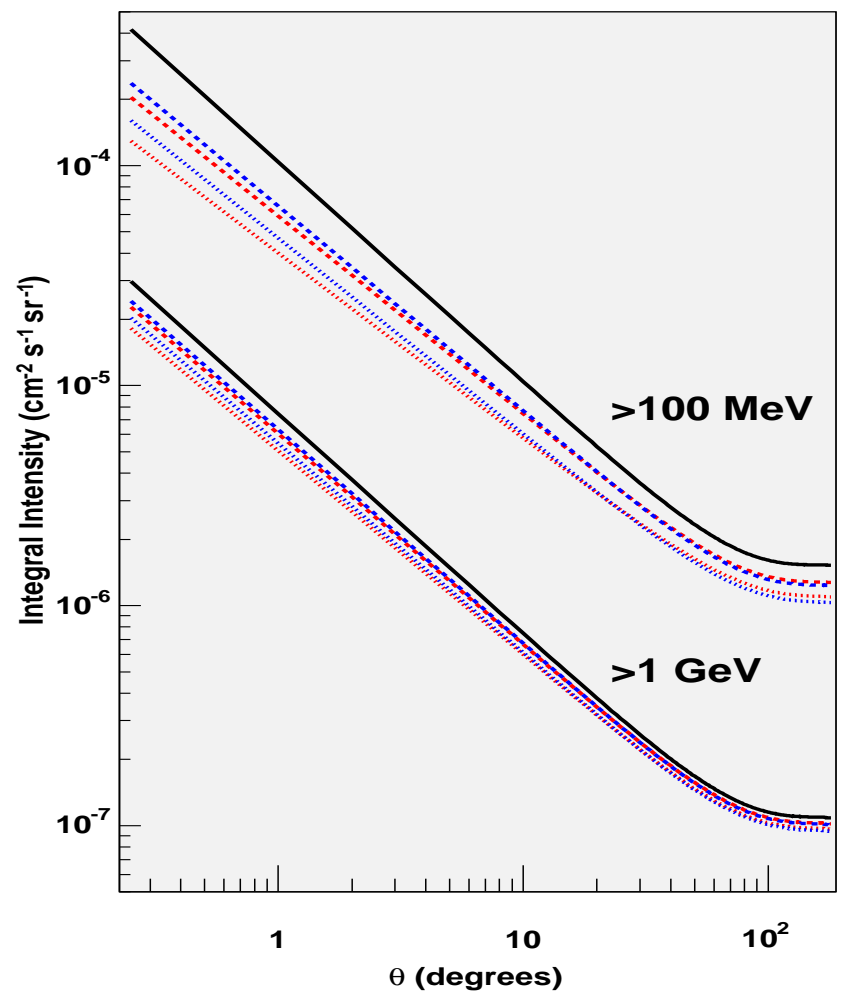

FIG. 3.- Integral intensity above $100 \mathrm{MeV}$ and $1 \mathrm{GeV}$. Black line: no modulation; red lines: potential $\Phi_{1}$; blue lines: potential $\Phi_{2}$. Dashed lines: $\Phi_{0}=500 \mathrm{MV}$; dotted lines: $\Phi_{0}=1000 \mathrm{MV}$.

to the solar minimum and maximum conditions, respectively. There is a weak dependency on the modulation potential parameterisation. However, there is a much stronger dependency on $\Phi_{0}$ and thus the emission will vary over the solar cycle.

The FWHM of the EGRET point spread function is $5^{\circ}$ at $100 \mathrm{MeV}$. For $\theta<2.5^{\circ}$, we calculate an integral flux $F_{\gamma}(>100 \mathrm{MeV}) \sim 4 \times 10^{-7} \mathrm{~cm}^{-2} \mathrm{~s}^{-1}$ (modulated). The sum of the IC and albedo emission (Seckel et al. 1991) over this region is consistent with the EGRET upper limit of $2 \times 10^{-7} \mathrm{~cm}^{-2} \mathrm{~s}^{-1}$ (Thompson et al.1997), where the exact comparison would require extensive modelling which is beyond the scope of the present work.

The differential intensities for selected angles $\theta$ are shown in Fig. [4 Also shown is the extragalactic $\gamma$-ray background (EGRB) obtained by Strong at al. (2004), which includes true diffuse emission and an unresolved source component. Within $\theta \sim 10^{\circ}$ of the Sun, the solar IC emission is at least comparable to the EGRB. Table 11 gives the integral intensities above $10 \mathrm{MeV}, 100 \mathrm{MeV}$, and $1 \mathrm{GeV}$ averaged over the whole sky. The values for $500 \mathrm{MV}$ and $1000 \mathrm{MV}$ are obtained by averaging over the fluxes for $\Phi_{1}$ and $\Phi_{2}$. Above $100 \mathrm{MeV}$, the sky-averaged intensity is $\sim 20 \%$ of the EGRB obtained by Strong at al. (2004).

Note that unresolved blazars are thought to be the major contributors to the EGRB (Salamon \& Stecker 1998; Kneiske \& Mannheim 2005); thousands of them will be resolved by GLAST. Therefore, the currently derived EGRB flux is an upper limit on the true diffuse emission.
Table 1. All-Sky aVerage integral flux

\begin{tabular}{cccc}
\hline \hline & \multicolumn{1}{c}{$\Phi_{0}=0$} & $500 \mathrm{MV}$ & $1000 \mathrm{MV}$ \\
\hline$>10 \mathrm{MeV}$ & 17.8 & 10.5 & 7.5 \\
$>100 \mathrm{MeV}$ & 2.2 & 1.8 & 1.5 \\
$>1 \mathrm{GeV}$ & 0.16 & 0.15 & 0.14 \\
\cline { 2 - 4 } Note. - Flux units & $10^{-6} \mathrm{~cm}^{-2} \mathrm{~s}^{-1} \mathrm{sr}^{-1}$.
\end{tabular}

\section{PERSPECTIVES FOR GLAST}

The Large Area Telescope (LAT) high-energy $\gamma$-ray detector, in preparation for launch by NASA in late 2007, will have unprecedented angular resolution, effective area, and field of view for high-energy $\gamma$-rays (McEnerv et al. 2004). The LAT will scan the sky continuously and provide essentially complete sky coverage every 2 orbits (approximately 3 hours). As a consequence, the light curve for any detectable source will be well sampled. Also, the scanning motion will permit frequent evaluation of the stability of the performance of the LAT with respect to "standard candle" sources. Based on the expected sensitivity of the $\mathrm{LAT}^{2}$, a source with flux $\sim 10^{-7} \mathrm{~cm}^{-2} \mathrm{~s}^{-1}$ and the hardness of the solar IC emission will be detectable on a daily basis when the Sun is not close to the Galactic plane, where the diffuse emission is brightest. Sensitive variability studies of the bright core of the IC emission surrounding the Sun should be possible on weekly time scales. With exposure accumulated over several months, the Sun should be resolved as an extended source and potentially its IC and pion decay components separated spatially.

The extended IC emission is not isotropic, but accumulated over year timescales will be uniform in ecliptic longitude and brightest at low ecliptic latitudes. The ecliptic plane crosses the Galactic plane near the Galactic Centre, and the solar IC component may be important for investigating the nature of the reported "halo" of $\gamma$-rays about the Galactic Centre (Dixon et al. 1998).

The solar IC contribution to the overall celestial diffuse emission can be modelled directly as we have done here. Measurement of the spectrum of solar IC emission in the near Sun direction fixes the emission at all angles. This is especially true at high energies where the solar modulation in the outer heliosphere is negligible. Also, for any particular direction of the sky the Galactic and extragalactic components can be derived from the data by measuring variations in diffuse intensity with solar elongation angle.

\section{DISCUSSION AND CONCLUSION}

In this paper we have studied the IC scattering of solar photons by CR electrons. We have shown that the emission is significant and broadly distributed with maximum brightness in the direction of the Sun. The whole sky is shining in $\gamma$-rays contributing to a foreground that would otherwise be ascribed to the Galactic and extragalactic diffuse emission.

The IC emission from CR electrons depends on their spectrum in the heliosphere and varies with the modulation level. Observations in different directions can be

2 http://www-glast.slac.stanford.edu 

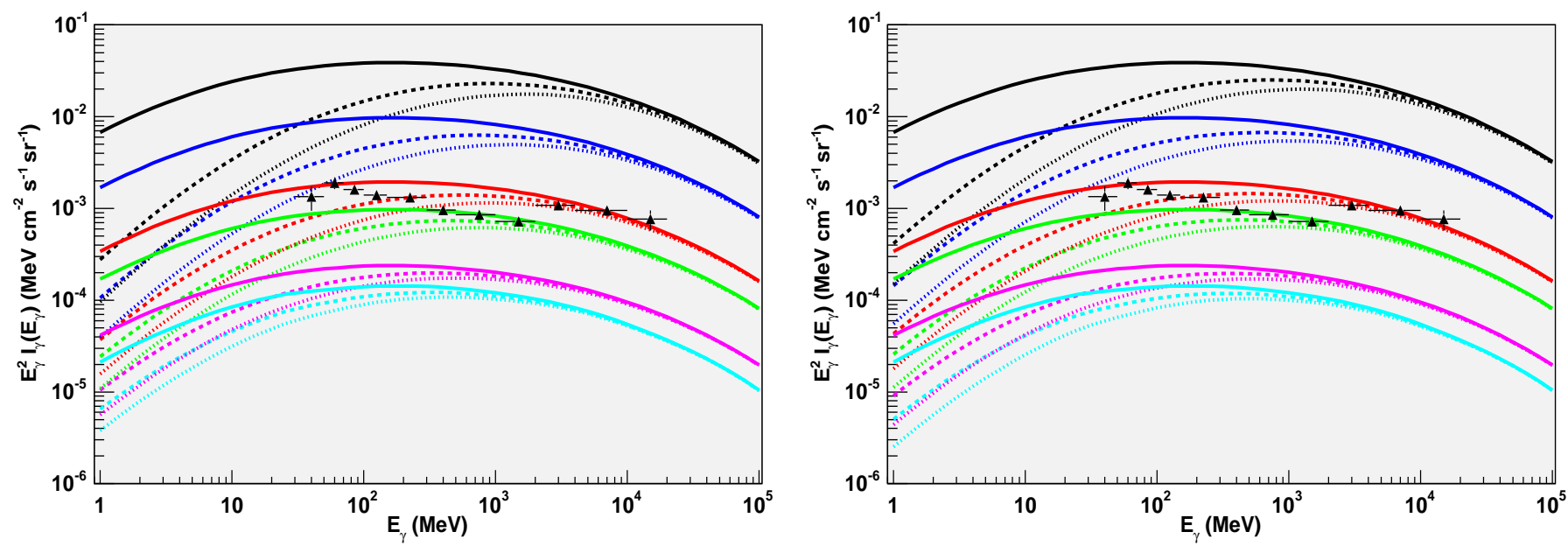

FIG. 4.- Differential intensities for selected $\theta$. Line-sets (top to bottom): $\theta \sim 0.25^{\circ}, 1^{\circ}, 5^{\circ}, 10^{\circ}, 45^{\circ}$, and $180^{\circ}$. Solid line: no modulation; dashed line: $\Phi_{0}=500 \mathrm{MV}$; dotted line: $\Phi_{0}=1000 \mathrm{MV}$. Left panel: $\Phi_{1}$ modulation potential; right panel: $\Phi_{2}$ modulation potential. Data points: diffuse extragalactic $\gamma$-ray flux (Strong at al. 2004).

used to determine the electron spectrum at different heliocentric distances. A sensitive $\gamma$-ray telescope on orbit could monitor the heliosphere providing information on its dynamics. Such observations can also be used to study the electron spectrum in close proximity to the Sun, unreachable for direct measurements by spacecraft.

What are the immediate implications? Since solar modulation theory is well-developed (e.g., Ferreira \& Potgieter 2004), accurate measurement of the electron spectrum near the solar surface will open the way to derive the spectra of other Galactic CR species, primarily protons, near the Sun. The CR proton spectrum is the input to calculations for spectral predictions of $\gamma$-rays from $p p$-interactions in the solar atmosphere (Seckel et al. 1991). These can be further tested using GLAST observations of pionic $\gamma$-rays from the Sun. In turn, this would provide information about the density profile of the solar atmosphere, magnetic field(s), and CR cascade development. The higher the energy of $\gamma$-rays, the higher the energy of the ambient particles, and thus the depth of the layers tested is increased. In conjunction with other solar monitors this can bring understanding of the deep atmospheric layers, Sun spots, magnetic storms, and other solar activity.

Furthermore, understanding of the solar atmosphere is necessary to calculate the neutrino flux from $p p$ - interactions at higher energies $(>1 \mathrm{TeV})$ as the $\mathrm{CR}$ shower development depends on the density distribution and the underlying magnetic field structure. Finally, the "calibrated" neutrino flux from the Sun can be used as a "standard candle" for $\mathrm{km}^{3}$ neutrino telescopes of the near future, such as IceCube (e.g., Hettlage et al. 2000; Ahrens et al. 2004). Furthermore, the solar core is opaque for VHE neutrinos (Seckel et al. 1991; Moskalenko \& Karakula 1993; Ingelman \& Thunman 1996) thus observations of the neutrino flux, or rather its deficit, can provide us with information about the solar mass distribution.

To summarise, we have shown that the observation of the IC emission from CR electrons in the heliosphere and its distribution on the sky will open a new chapter in astrophysics. This makes a sensitive $\gamma$-ray telescope a useful tool to monitor the heliosphere and heliospheric propagation of $\mathrm{CR}$, to study $\mathrm{CR}$ interactions in the solar atmosphere, and even to calibrate VHE neutrino telescopes enabling them to look deep into the solar core.

I. V. M. acknowledges partial support from NASA Astronomy and Physics Research and Analysis Program (APRA) grant. T. A. P. acknowledges partial support from the US Department of Energy.

\section{REFERENCES}

Ahrens, J., et al., 2004, New Astron. Rev., 48, 519

Dixon, D. D., et al., 1998, NewA., 3, 539

Ferreira, S. E. S., \& Potgieter, M. S., 2004, ApJ, 603, 744

Fujii, Z., \& McDonald, F. B., 2005, Adv. Space Res., 35, 611

Gleeson, L. J., \& Axford, W. I., 1968, ApJ, 154, 1011

Hettlage, C., Mannheim, K., \& Learned, J. G., 2000, APh, 13, 45

Ingelman, G. \& Thunman, M., 1996, Phys. Rev. D, 54, 4385

Kneiske, T. M., \& Mannheim, K., 2005, in Proc. 2nd Int. Symp. on High Energy Gamma-Ray Astronomy, ed. F. A. Aharonian et al. (New York: AIP), AIP Conf. Proc., v.745, 578

Livingston, W. C., 1999, in Allen's Astrophysical Quantities, ed. A. N. Cox (4th ed.; New York: Springer), 339

McEnery, J. E., Moskalenko, I. V., \& Ormes, J. F., 2004, in Cosmic Gamma-Ray Sources, eds. K. S. Cheng \& G. E. Romero (Dordrecht: Kluwer), Astrophys. \& Spa. Sci. Library, v.304, 361
Moskalenko, I. V., Karakula, S., \& Tkaczyk, W., 1991, A\&A, 248, L5

Moskalenko, I. V., \& Karakula, S., 1993, J. Phys. G: Nucl. Part. Phys., 19, 1399

Moskalenko, I. V., \& Strong, A. W., 2000, ApJ, 528, 357

Ptuskin, V. S., Moskalenko, I. V., Jones, F. C., Strong, A. W., \& Zirakashvili, V. N., 2006, ApJ, 642, 902

Salamon, M. H., \& Stecker, F. W. 1998, ApJ, 493, 547

Seckel, D., Stanev, T., \& Gaisser, T. K., 1991, ApJ, 382, 652

Strong, A. W., Moskalenko, I. V., \& Reimer, O., 2004, ApJ, 613, 956

Thompson, D. J., Bertsch, D. L., Morris, D. J., \& Mukherjee, R., 1997, J. Geophys. Res. A, 120, 14735 